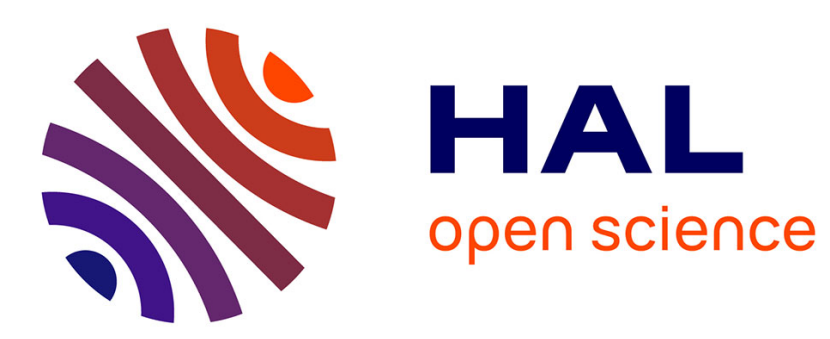

\title{
Toward a real time SIMO channel emulator
}

\author{
Paul Chatelier, Patrick Tortelier, Emmanuel Boutillon
}

\section{To cite this version:}

Paul Chatelier, Patrick Tortelier, Emmanuel Boutillon. Toward a real time SIMO channel emulator. WIMOB 2007, 3rd IEEE int. conf. on Wireless and Mobile Computing, Networking and Communications, New York, USA, Oct. 2007, Oct 2007, New York, United States. pp.Clef USB. hal-00450745

\section{HAL Id: hal-00450745 \\ https://hal.science/hal-00450745}

Submitted on 26 Jan 2010

HAL is a multi-disciplinary open access archive for the deposit and dissemination of scientific research documents, whether they are published or not. The documents may come from teaching and research institutions in France or abroad, or from public or private research centers.
L'archive ouverte pluridisciplinaire HAL, est destinée au dépôt et à la diffusion de documents scientifiques de niveau recherche, publiés ou non, émanant des établissements d'enseignement et de recherche français ou étrangers, des laboratoires publics ou privés. 


\section{Toward a hardware real time SIMO channel emulator}

\author{
Paul Chatellier \\ France Telecom \\ Email: paul.chatellier@orange-ftgroup.fr
}

\author{
Patrick Tortelier \\ France Telecom \\ Issy les Moulineaux, France
}

\author{
Emmanuel Boutillon \\ LESTER, University of South Brittany \\ Lorient, France
}

\begin{abstract}
Experience shows that the fading channel is one of the most time consuming element of a wireless system simulation, especially when dealing with multiple antennas. Any method that reduces this simulation time is thus welcomed. As an interesting solution, real time simulation using an hardware platform is the most commonly used, especially with the help of high level synthesis tools which will shrink the gap between system level simulations, written in $\mathrm{C}$ or $\mathrm{C}++$, and hardware level. In this paper, we extend an existing model of Rayleigh faders with interesting complexity to a SIMO case. We show, analytically and by simulation, that the spatial correlation of our simplified channel model are in accordance with the expected one. For a more realistic model, we take into account the existence of angle spread scenarii.
\end{abstract}

\section{INTRODUCTION}

In the past few years MIMO systems have gained a considerable attention due to the expected increase in data rates they promise [1]. Actually MIMO channel capacity is known to increase linearly with the rank $K$ of its matrix [2]. For a $N_{T}$ (transmitter antennas) by $N_{R}$ (receiver antennas) MIMO channel, rank $K$ is given by

$$
K \leq \min \left(N_{T}, N_{R}\right)
$$

New communication schemes involving MIMO systems are not only regularly published but also normalized (WiMax, for example). The MIMO schemes are more and more efficient and complex, especially when they use some form of iterative decoding (turbo principle). From a designer perspective, the development and the optimisation of a receiver architecture is a very complex problem. In fact, their performance cannot be assessed with closed form formulas and Monte-Carlo simulations are mandatory to evaluate performances. Unfortunately, Monte-Carlo simulations are very time consuming. For example, the evaluation of a BER of 10-6 requires the simulation of $10^{9}$ bits. Using a classical software simulation, the estimation of such a BER may require hours of simulation. An alternative solution is to use hardware emulation in order to have bit rate throughput closed or equal to the real time system and thus, reduced time of simulation. In order to perform this type of hardware simulation, a MIMO channel emulator should also be used. Thus, we focus our attention on channel hardware emulation. In this paper, a first step toward MIMO hardware emulator is done by investigating a new SIMO channel simulator under constraint of hardware implementation in order to make the development of the equivalent emulator easier.

A predominant property of SIMO channels is the spacetime correlation introduced in transmission, which heavily influences the design of transmitted waveforms and receiver algorithms to mitigate the effects of correlated fading. Time correlation has long been taken into account, Jake's model [3] and its variations [4]-[7] being commonly used in simulations for SISO channels. Some other work address the problem of computational load of these algorithms, particularly [8] where the sum of sinusoids is simplified by sum of finite triangular waves.

For more realistic channel model, angle spread has to be taken into account. In fact angle spread ensues from selected environment (indoor/outdoor, urban, ... ) and changes spatial correlation of the received signal. Typically, in a macrocell environment, the base station is deployed higher than the surrounding scatterers. Hence, the multipath components at the base station are restricted to a smaller angular distribution. This change in the behavior of the received signal is very important as far as antenna array applications are concerned.

Algorithms to introduce spatial correlation in a MIMO channel have been derived, see for instance the 3GPP spatial MIMO channel model [9]. The method used leans on the fact that waves incoming to receiver can be considered as plane waves, allowing the computation of spatial correlation between antenna elements by introducing phase lags of each plane wave between these antennas. The plane wave approximation can be done when assumption of far field is assumed. It means that distances between transmitter and the different antennas of the receiver are approximatively the same. Differences between these distances must be largely inferior to one wavelength so differences of phase are largely inferior to $\pi$. The Fraunhaufer condition of far field defines a minimal distance $d_{\text {frau }}$ between transmitter and receiver

$$
d>d_{\text {frau }}=\frac{2 D^{2}}{\lambda_{c}} \quad \text { and } \quad d>>\lambda_{c}
$$

where $d$ is the distance between transmitter and receiver, $D$ is the distance spatial evaluation zone of the wave and $\lambda_{c}$ is the wavelength of the carrier frequency. Hence, for a zone of $3 \lambda_{c}$ sidelong (for example a linear network of 4 dipoles each spaced by $\left.\lambda_{c}\right), d_{\text {frau }}=2,7 \mathrm{~m}$ at $2 \mathrm{GHz}$. This result shows that plane wave assumption is good in most of cases. 
In Section II, the SISO channel model defined in [8] is introduced and some results on our hardware implementation are briefly presented. Then, we proposed, in Section III, SIMO extension of this algorithm, with considereration on spatial correlation between receiver antennas. Its spatial correlation function is theoretically investigated. In Section IV, the computer simulations process is described and results on the spatial correlation function of the experimental model are presented. Finally, the Section V concludes this paper.

\section{Y. Li AND X. HuANG FADing Generator [8]}

In this section, we pay attention on one particular model of multiple independent Rayleigh faders satisfying the above outlined conditions. First, we introduce the model. Then, having in mind hardware emulation, we briefly present some results on our hardware implementation.

\section{A. Model definition}

Deterministic models of rayleigh faders are derived from central limit theorem. Thus a Gaussian process can be approximated by a large number of properly weighted sinusoids with uniformly distributed phases. Considering $M$ fading waveforms, each of which composed of $N_{s p}$ sinusoids, the $p^{\text {th }}$ fading waveform expression is

$$
h_{p}(t)=\sum_{s=0}^{N_{s p}-1} \alpha_{p, s} e^{j\left(\omega_{p, s} t+\varphi_{p, s}\right)}, \quad p=0,1, \cdots, M-1
$$

where $\alpha_{p, s}, \omega_{p, s}$ and $\varphi_{p, s}$ represent the amplitude, the Doppler frequency shift and the uniformly distributed phases of the sth complex sinusoid in the $p$ th fader. $\omega_{p, s}$ depends on maximum Doppler frequency $\omega_{M}=2 \pi v / \lambda_{c}$ (where $v$ is the velocity of receiver and $\lambda_{c}$ is the wavelength of the transmitted carrier frequency) and sinusoid Angle Of Arrival (AOA) $\theta_{p, s}$ by the relation

$$
\omega_{p, s}=\omega_{M} \cos \theta_{p, s}
$$

By assuming an asymmetrical distribution of angle of arrival and defining a particular symmetry in $\varphi_{p, s}, \mathrm{Y} . \mathrm{Li}$ and $\mathrm{X}$. Huang proposed a model that fits better the desired Rayleigh fading waveform with only $N_{0}=N_{s p} / 4$ rays needed. Then, the authors proposed an interesting simplification of their model which leans on replacement of sinusoids by finite triangular wave. Hence, the reduction of the complexity is very important and an the model becomes very simple. Even generator based on lookup table approximation of sinusoids can not compete with.

Therefore this model is suitable for simulation purpose but also for hardware implementation thanks to its simplicity. Currently, this last point is expanding because of the time to market pressure. In fact, hardware simulator can speed up simulation to real-time and enhance algorithm validation by taking into account limitation of resources due to its final environment (for example, width of the internal variable).

\section{B. Hardware implementation}

Particularly, one of our research center of interest is use of Field Programmable Gate Array (FPGA) and High Level Synthesis (HLS) tools for the development of digital communication algorithms. Hence hardware design explanation is done by tools from a simple behavioral description. Thus algorithms coded in $\mathrm{C} / \mathrm{C}++$ (for example) are reused to generate hardware designs.

Here, a C++ behavioral description of the SISO channel model is given (see fig. 1) as input of HLS tool CatapultC [10].

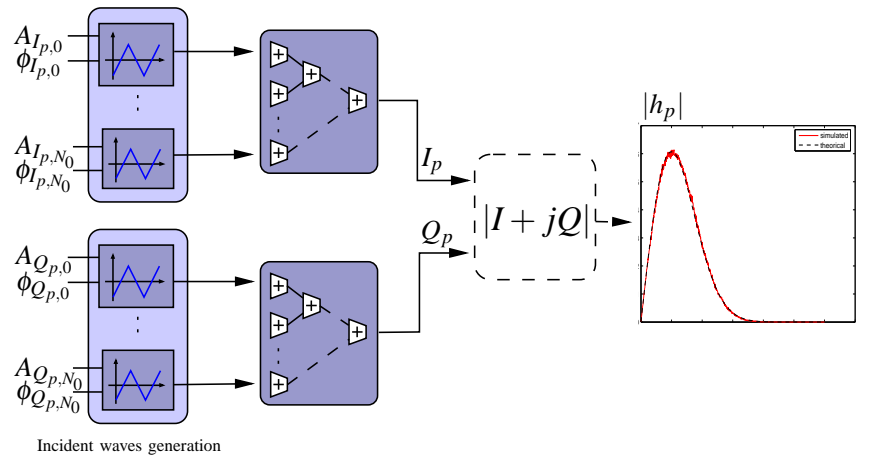

Fig. 1. Architecture of the $p$ th path of the SISO channel simulator. $A_{I_{p}, n}$ and $\phi_{I_{p}, n}$ are, respectively, the slope and the origin of triangular waves for the inphase component. Identically, $A_{Q_{p}, n}$ and $\phi_{Q_{p}, n}$ for the quadrature component.

The hardware target is the small FPGA APEX20KE15003 of Altera with a working frequency fixed to $25 \mathrm{MHz}$ (in relation with our hardware platform). The emulator must provide an output at each top of the clock. Thus, the HLS tool is configured to achieve an architecture on this target that fit a throughput of $25 \mathrm{Mbits} / \mathrm{s}$.

The generated design is validated by comparing the theoretical temporal correlation function with the experimental one (issues from hardware simulation). Results are presented in figure (2). For theoretical demonstration, readers can refer to [8].

Briefly, some remarks on this design herited from hardware information are presented on Table I for different number $M$ of faders.

\begin{tabular}{|c|c|c|c|c|}
\hline$M$ & $N_{s p}$ & Throughput & Area (\%) & Area ratio \\
\hline \hline 1 & 8 & $25 \mathrm{Mbit} / \mathrm{s}$ & 11 & $(\times) 1.00$ \\
\hline 4 & 8 & $25 \mathrm{Mbit} / \mathrm{s}$ & 43 & $(\times) 3.91$ \\
\hline 8 & 8 & $25 \mathrm{Mbit} / \mathrm{s}$ & 86 & $(\times) 7.82$ \\
\hline
\end{tabular}

TABLE I

HARDWARE ARCHITECTURE FOR DIFFERENT SCENARII.

First, we note that the area increases linearly with the number of paths considered. Hence, the HLS tool realized an automatic explanation of possible architecture involving the selection of a parralel architecture which satisfy our constraint. Another important result is the very small area required for our design. By changing these parameters, the hardware block 


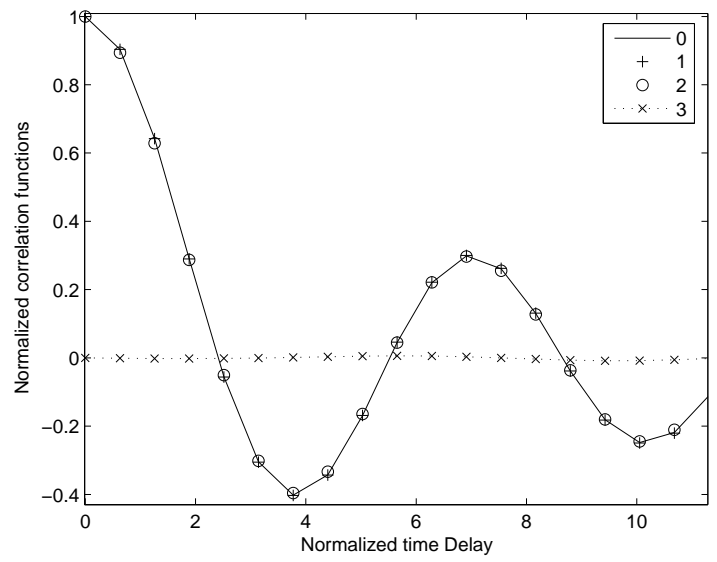

Fig. 2. Correlation function of the Y. Li and X. Huang model with parameters $M=8$ and $N_{0}=8$. 0 : theoretical autocorrelation; 1 : autocorrelation of fader 0 generated by sum-of-sinusoids model; 2: autocorrelation of fader 0 generated with sum of finite triangular waves; 3: cross correlation function of fader 0 and fader 4 (hardware simulation). The normalized time delay is defined as $\omega_{M} \tau$.

defined above can be used for different distribution of AOAs and even different models. That is what we do in the next section where our extension to a SIMO channel model is studied.

\section{AN EXTENDED MODEL FOR SIMO CHANNEL SIMULATOR}

Since interest of finite triangular waves interpolation is demonstrated, it can be used for more complex model like SIMO channel. By construction, the above Rayleigh fading generator works only for AOA uniformly distributed in $[0,2 \pi[$. For a more realistic channel simulator, different AS have to be considered. Actually, the addition of plane-waves causes space selective fading at the receive antennas. The resulting fading correlation is governed by the angle spread, the antenna spacing $d_{s}$, and the wavelength. Assuming uniform linear array (ULA) of antennas, the spatial correlation function depends on propagation time delay between sub-path of each antenna (see fig. (3)).

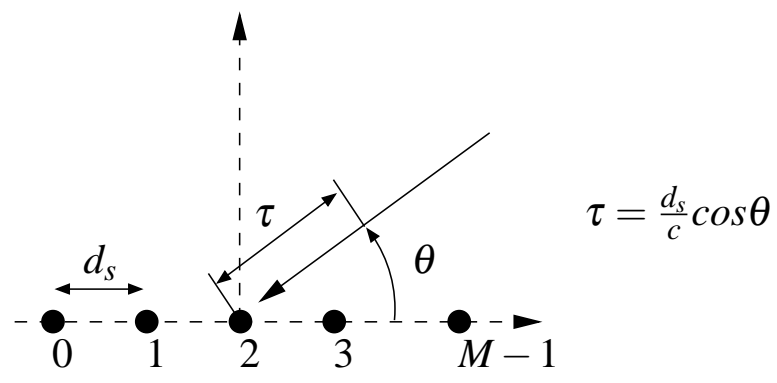

Fig. 3. Uniform Linear Array ( $c$ is the velocity of light)

Taking the element 0 as reference, the array response of a ULA is given by

$$
a(\theta)=\left[1, \ldots, e^{j 2 \pi m \frac{d_{S}}{\lambda_{c}} \cos \theta}, \ldots, e^{j 2 \pi(M-1) \frac{d_{S}}{\lambda_{c}} \cos \theta}\right]
$$

Hence, a spatial model can be defined by the combination of (3) and (5)

$$
h_{p, m}(t)=\sum_{s=0}^{N_{s p}-1} \alpha_{p, s} e^{j\left(\omega_{p, s} t+\varphi_{p, s}\right)} e^{j 2 \pi m \frac{d_{S}}{\lambda_{c}} \cos \theta}
$$

Several distribution can be considered for the direction of arrival for each multi-path components, including uniform, Gaussian, Laplacian, etc. For the sake of simplicity, we consider, here, one path with uniformly distributed sub-paths over $\left[\bar{\theta}-\Delta_{\theta} ; \bar{\theta}+\Delta_{\theta}\right]$ with equal power. Thereafter, we define the Power Angular Spectrum (PAS) $\rho_{A S}(\theta)$ as

$$
\rho_{A S}(\theta)= \begin{cases}\frac{\Omega}{2 \Delta_{\theta}} & \text { with } \theta \in\left[\bar{\theta}-\Delta_{\theta} ; \bar{\theta}+\Delta_{\theta}\right] \\ 0 & \text { elsewhere }\end{cases}
$$

where $\Omega$ is the total power of all sub-paths. The normalized spatial correlation function is computed as

$$
\Gamma\left(d_{S}\right)=E\left\{h_{p, m}(t, r) h_{p, m+1}^{*}(t, r+d s)\right\}
$$

where $r$ is the position of the $m^{\text {th }}$ antenna. Combining the three equations above yields well-known [11] normalized theoretical spatial correlation $\Gamma_{\text {norm }}\left(d_{S}\right)=\Gamma\left(d_{S}\right) / \Gamma(0)$ (calculated for an infinite number of sub-paths $\left.\left(N_{s p} \rightarrow+\infty\right)\right)$ expressed below

$$
\begin{aligned}
\Re\left[\Gamma_{\text {norm }}\left(d_{S}\right)\right]= & J_{0}\left(2 \pi \frac{d_{S}}{\lambda_{c}}\right)+2 \sum_{k=1}^{+\infty}(-1)^{k} J_{2 k}\left(2 \pi \frac{d_{S}}{\lambda_{c}}\right) \\
& \cdot \cos (2 k \bar{\theta}) \operatorname{sinc}\left(k \Delta_{\theta_{\max }}\right) \\
\mathfrak{I}\left[\Gamma_{\text {norm }}\left(d_{S}\right)\right]= & -2 \sum_{k=0}^{+\infty}(-1)^{k} J_{2 k+1}\left(2 \pi \frac{d_{S}}{\lambda_{c}}\right) \\
& \cdot \cos (2 k \bar{\theta}) \operatorname{sinc}\left(k \Delta_{\theta_{\max }}\right)
\end{aligned}
$$

where $\Delta_{\theta_{\max }}=2 \Delta_{\theta}$. Simplifying equation 6 by use of finite triangular waves instead of sinusoids will reduce implementation complexity. This simplification does not degrade temporal correlation function in a SISO case. Hence, we calculate the theoretical spatial correlation function obtained in a SIMO case proving that SISO case can be extended to SIMO with consideration on AS.

Triangular waves can be described by their Fourier development

$$
f(\theta)=\frac{-4 \sqrt{6}}{\pi^{2}} \sum_{n=0}^{+\infty} \frac{\cos (2 n+1) \theta}{(2 n+1)^{2}}
$$

By replacing complex exponential in (6) by (10), the channel response evolves in 


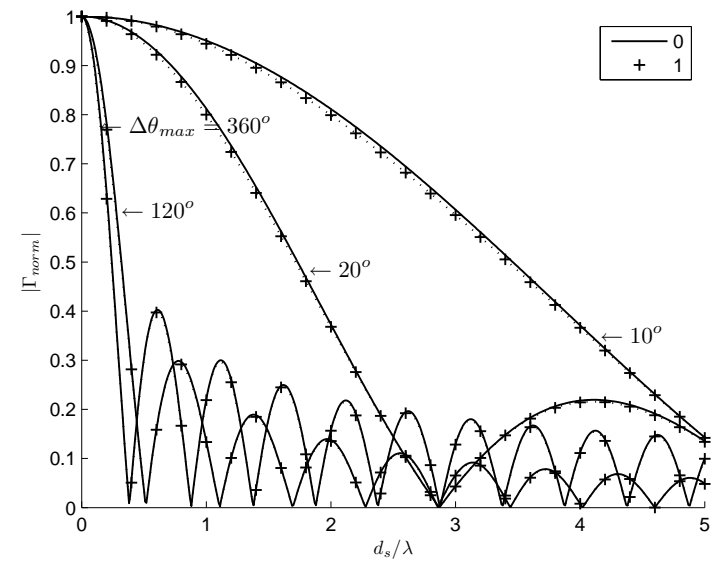

(a) $\bar{\theta}=90^{\circ}$

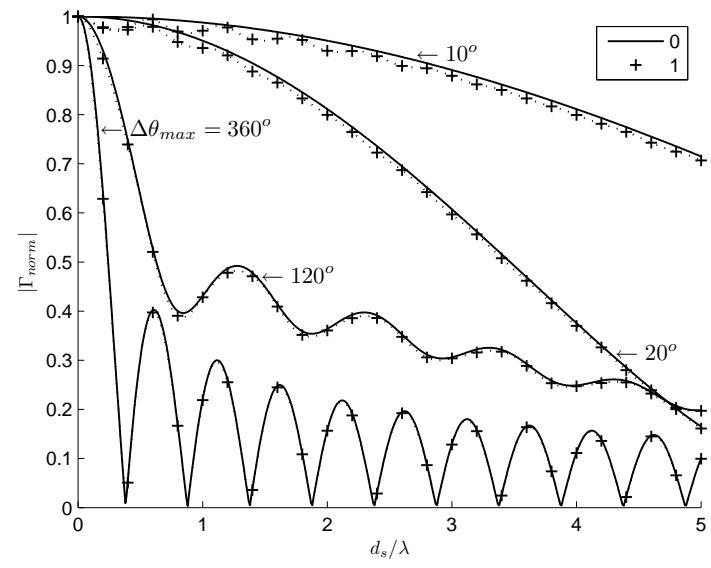

(b) $\bar{\theta}=30^{\circ}$

Fig. 4. Theoretical spatial correlation of one path of the low complexity SIMO channel model. 0: analytical spatial correlation of the sum-of-sinusoids model; 1: analytical spatial correlation of the sum of finite triangular waves model

$$
\begin{aligned}
h_{p, m}= & \frac{-4 \sqrt{6}}{\pi^{2}} \sum_{s=0}^{N_{s p}-1} \sum_{n=0}^{+\infty} \frac{\alpha_{p, s}}{(2 n+1)^{2}} \\
& \left(\cos \left[(2 n+1)\left(\omega_{p, s} t+\varphi_{p, s}+\phi_{p, s}(m)\right)\right]+\right. \\
& \left.j \sin \left[(2 n+1)\left(\omega_{p, s} t+\varphi_{p, s}+\phi_{p, s}(m)\right)\right]\right)
\end{aligned}
$$

where $\phi_{p, s}(m)=2 \pi m \frac{d_{S}}{\lambda_{c}} \cos \theta_{p, s}$ is the phase lag between antennae of reference and $m$ th antennae for the $s^{\text {th }}$ ray of the $p^{\text {th }}$ path.

Assuming a uniformly distributed phases $\varphi_{p, s}, \Gamma\left(d_{S}\right)$ only depends on $\phi_{p, s}(m)$. Moreover, with PAS defined on equation (7) and an infinite number of sub-paths, the normalized spatial correlation function can be written in its integral form

$$
\begin{aligned}
\Gamma_{\text {norm }}\left(d_{S}\right) & =\frac{\Gamma\left(d_{S}\right)}{\Gamma(0)} \\
& =\frac{1}{2 \Delta_{\theta}} \int_{\bar{\theta}-\Delta_{\theta}}^{\bar{\theta}+\Delta_{\theta}} \frac{1}{(2 n+1)^{2}} \times \\
& \left(\cos \left[(2 n+1)\left(\phi_{p, s}(m)\right)\right]+\right. \\
& \left.j \sin \left[(2 n+1)\left(\phi_{p, s}(m)\right)\right]\right) d \theta
\end{aligned}
$$

Using the two following relations defined in [12]

$$
\begin{aligned}
& \cos (z \cos (x))=J_{0}(z)+2 \sum_{k=1}^{+\infty}(-1)^{k} J_{2 k}(z) \cos (2 k x) \\
& \sin (z \cos (x))=-2 \sum_{k=0}^{+\infty}(-1)^{k} J_{2 k+1}(z) \cos ((2 k+1) x)
\end{aligned}
$$

and the integral development

$$
\frac{1}{2 \Delta_{\theta}} \int_{\bar{\theta}-\Delta_{\theta}}^{\bar{\theta}+\Delta_{\theta}} \cos (a \theta) d \theta=\cos (a \bar{\theta}) \operatorname{sinc}\left(2 k \Delta_{\theta}\right)
$$

equation (11) yields following expression for the real and imaginary part of $\Gamma_{\text {norm }}\left(d_{S}\right)$

$$
\begin{gathered}
\mathfrak{R}\left[\Gamma_{\text {norm }}\left(d_{S}\right)\right]=\frac{96}{\pi^{4}} \sum_{n=0}^{+\infty} \frac{1}{(2 n+1)^{4}} \\
\left\{J_{0}\left((2 n+1) 2 \pi \frac{d_{S}}{\lambda_{c}}\right)+2 \sum_{k=1}^{+\infty}(-1)^{k} \times\right. \\
\left.J_{2 k}\left((2 n+1) 2 \pi \frac{d_{S}}{\lambda_{c}}\right) \cos (2 k \bar{\theta}) \operatorname{sinc}\left(2 k \Delta_{\theta}\right)\right\} \\
\mathfrak{I}\left[\Gamma_{\text {norm }}\left(d_{S}\right)\right]=\frac{96}{\pi^{4}} \sum_{n=0}^{+\infty} \frac{-2}{(2 n+1)^{4}} \sum_{k=1}^{+\infty}(-1)^{k} \\
J_{2 k+1}\left((2 n+1) 2 \pi \frac{d_{S}}{\lambda_{c}}\right) \cos ((2 k+1) \bar{\theta}) \\
. \operatorname{sinc}\left((2 k+1) \Delta_{\theta}\right)
\end{gathered}
$$

Figure 4 presents the magnitude of $\Gamma_{\text {norm }}$ for different mean angles of incidence and angle spreads. There is no discrepancy between correlation function obtained for each scenario. Note that in the case of uniformly distributed phases over $[0 ; 2 \pi[$, curves merge together. It leans on the fact that expression (13) of $\Gamma\left(d_{s}\right)$ is reduced to

$$
\Gamma_{n o r m}\left(d_{S}\right)=\frac{96}{\pi^{4}} \sum_{n=0}^{+\infty} \frac{1}{(2 n+1)^{4}} J_{0}\left((2 n+1) 2 \pi \frac{d_{S}}{\lambda_{c}}\right)
$$

which is a good approximation of $J_{0}\left(2 \pi \frac{d_{S}}{\lambda_{c}}\right)$.

\section{Simulations}

It must be kept in mind that the theoretical spatial correlation obtained above assumes an infinite number of subpaths. As we are interested in a real implementation of this channel model me must take into account that we can only use a finite number of subpaths. An analytical derivation is no longer possible, and a simulation is the only convenient way to assess the behaviour of the model under the assumption of a finite number of rays, usually $N_{s p} \approx 20$. 


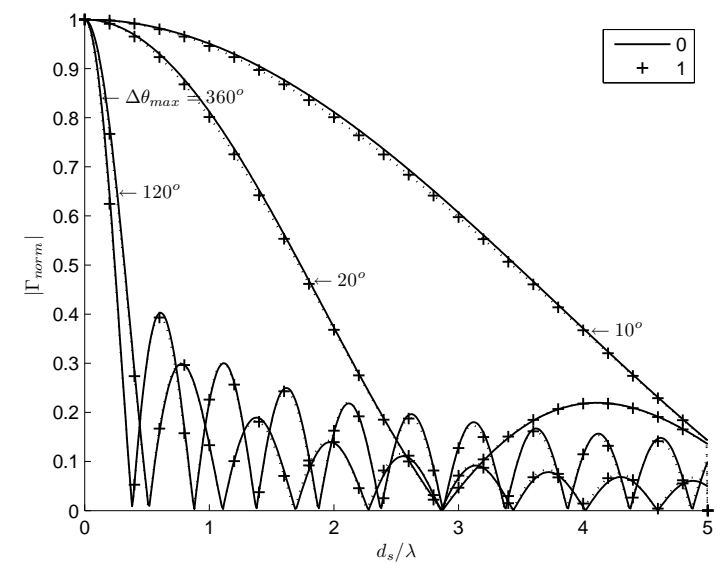

(a) $\bar{\theta}=90^{\circ}$

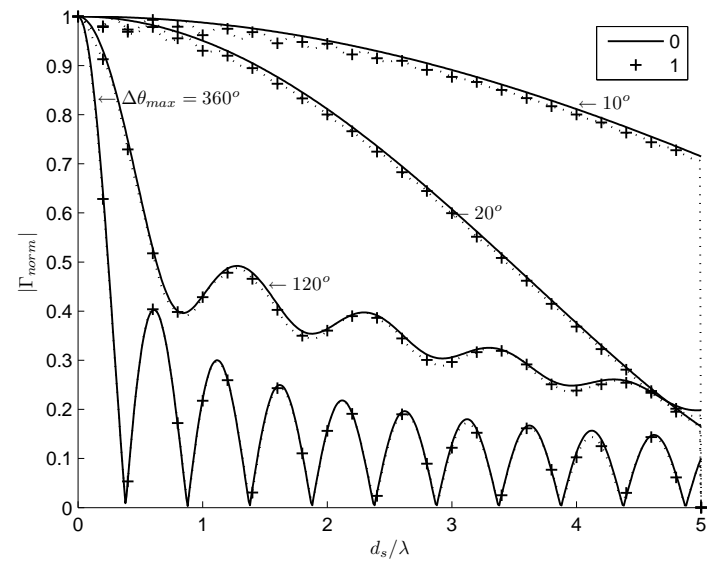

(b) $\bar{\theta}=30^{\circ}$

Fig. 5. Experimental spatial correlation of one path of the low complexity SIMO channel model generated for $N_{s p}=20$. 0: Analytical spatial correlation of the sum-of-sinusoids model; 1: obtained spatial correlation of the sum of finite triangular waves model.

we must also stress that in the process of derivation of the theoretical derivation leading to equations (9) and (13) we have used that the angles $\varphi_{p, s}$ (the phases of $p^{\text {th }}$ fader) are random quantities; therefore the simulation has to draw a number of different channel realizations (corresponding to different initial phases of the faders) in order to comply this assumption.

In order to take into account the finite number of subpaths simulations were performed for 20 subpaths. We observed that the simplification we used (triangle replacing sinusoid) does not cause any discrepancy with sum of sinusoids models; fig 5 shows spatial correlations obtained by simulation, for various angle spreads and mean angle of arrival; we observe very good agreement with the results of the sum of sinusoids model.

Furthermore, our simulation allowed us to take easily into account the case of non uniform distribution of sub-paths within the angle spread of subpaths, the observed spatial correlation can no longer be matched with theoretical results but are in good agreement with previous published results.

\section{CONCLUSION}

In this paper, we have introduced a SISO channel model issued from [8] and have proposed a reference hardware block for generation of correlated faders. Therefore, an extension to a SIMO channel simulator have been investigated and analytical spatial correlation function is defined. Simulations shows that with an acceptable number of rays this model give results that fit very good the expected theoretical spatial correlation function.

Besides those lines, we underlined our research on low complexity algorithm to enable the development of efficient channel emulators. Future works will be concerned with proposition of a SIMO hardware emulator by using the hardware block described above and extension of the SIMO model toward a MIMO model.

\section{REFERENCES}

[1] I. E. Telatar, "Capacity of multi-antennas gaussian channels," AT\&T Bell Labs Internal Memo., June 1995.

[2] G. J. Foschini and M. J. Gans, "On limits of wireless communications in a fading environment when using multiple antennas," Wireless Personnal Communications, vol. 6, no. 3, pp. 311-335, March 1998.

[3] W. C. Jakes, Microwave Mobile Communications, 1974.

[4] P. Dent, G. Bottomley, and T. Croft, "Jakes fading model revisited," Electronic Letters, vol. 29, no. 12, pp. 1162-1163, June 1993.

[5] Y. Li and Y. Guan, "Modified jakes model for simulating multiple uncorrelated fading waveforms," IEEE Transaction on Communications, vol. 50, no. 9, pp. 1503-1514, June 2000.

[6] M. Pop and N. Beaulieu, "Limitation of sum-of-sinusoids fading channel simulator," IEEE Transaction on Communications, vol. 49, pp. 699-708, April 2001.

[7] Y. R. Zheng and C. Xiao, "Simulation models with correct statistical properties for rayleigh fading channels," IEEE Transaction on Communications, vol. 51, no. 6, June 2003.

[8] Y. Li and X. Huang, "The Simulation of Independant Rayleigh Faders," IEEE Transaction on Communications, vol. 50, no. 9, September 2002.

[9] 3GPP, Universal Mobile Telecommunications System (UMTS) : Spatial channel model for Mulptiple Input Multiple output (MIMO) simulations, September 2003.

[10] Catapult C Synthesis C++ to Hardware Concepts, Mentor Graphics, Nov 2004.

[11] J. Salz and J. H. Winters, "Effect of fading correlation on adaptative arrays in digital mobile radio," IEEE transactions on Vehicular Technology, vol. 43, no. 4, november 1994.

[12] M. Abramowitz and I. A. Stegun, Handbook of Mathematical functions. Dover Publications, 1965. 